\title{
Predictive factors for invasive adenocarcinoma in patients with clinical non-invasive or minimally invasive lung cancer
}

\author{
Youngkyu Moon ${ }^{1}$, Jae Kil Park ${ }^{1}$, Kyo Young Lee ${ }^{2}$, Seha Ahn ${ }^{1}$, Jinwon Shin ${ }^{1}$ \\ ${ }^{1}$ Department of Thoracic \& Cardiovascular Surgery, ${ }^{2}$ Department of Hospital Pathology, Seoul St. Mary's Hospital, College of Medicine, The \\ Catholic University of Korea, Seoul, Republic of Korea \\ Contributions: (I) Conception and design: Y Moon; (II) Administrative support: JK Park; (III) Provision of study materials or patients: Y Moon, JK \\ Park, KY Lee; (IV) Collection and assembly of data: Y Moon, S Ahn, J Shin; (V) Data analysis and interpretation: Y Moon, KY Lee; (VI) Manuscript \\ writing: All authors; (VII) Final approval of manuscript: All authors. \\ Correspondence to: Youngkyu Moon, MD, PhD. Department of Thoracic \& Cardiovascular Surgery, Seoul St. Mary's Hospital, College of medicine, \\ The Catholic University of Korea, 222 Banpo-daero, Seocho-gu, Seoul 06591, Republic of Korea. Email: mykae@catholic.ac.kr.
}

\begin{abstract}
Background: Pure ground glass opacity (GGO) or part-solid GGO with small solid component $(\leq 5 \mathrm{~mm})$ are likely to be non-invasive or minimally invasive lung cancer. However, those lesions sometimes are diagnosed as invasive adenocarcinoma postoperatively. The aim of this study was to determine the predictors of invasive adenocarcinoma in clinical non- or minimally invasive lung cancer.

Methods: From January 2010 to December 2017, 203 patients were diagnosed as clinical adenocarcinoma in situ (AIS) or minimally invasive adenocarcinoma (MIA) identified on chest computed tomography (CT) and they underwent surgical resection. A retrospective study was performed to analyze the prediction of invasive adenocarcinoma in clinical non- or minimally invasive lung cancer.

Results: Of all clinical AIS or MIA patients, invasive adenocarcinoma was diagnosed in 55 patients $(27.1 \%)$. In clinical AIS, invasive adenocarcinoma was diagnosed in 19 patients (17.9\%) and 36 patients (37.1\%) were diagnosed as invasive adenocarcinoma in clinical MIA $(\mathrm{P}=0.002)$. Tumor diameter and the presence of solid component were confirmed to be significant predictive factors for invasive adenocarcinoma in a multivariate analysis [hazard ratio (HR) 1.071, $\mathrm{P}=0.037$; HR 2.573, $\mathrm{P}=0.005$; respectively].

Conclusions: Large tumor size and the presence of solid component in clinical AIS or MIA are predictive factors for invasive adenocarcinoma. Therefore, early surgical intervention is recommended for those lesions.
\end{abstract}

Keywords: Invasive adenocarcinoma; adenocarcinoma in situ (AIS); minimally invasive adenocarcinoma (MIA)

Submitted May 29, 2018. Accepted for publication Oct 09, 2018.

doi: $10.21037 /$ jtd.2018.10.83

View this article at: http://dx.doi.org/10.21037/jtd.2018.10.83

\section{Introduction}

Lung cancer is the fourth leading cause of cancer deaths in Korea. Recently, the use of chest computed tomography (CT) for lung cancer screening and early-stage lung cancer detection has increased (1), and with advances in technology, the detection of ground-glass opacity (GGO) has also increased remarkably. Persistent GGO is considered to be a precursor lesion of lung cancer or an early stage lung cancer $(2,3)$. However, surgical removal of all persistent GGO lesions is controversial. This is because many lesions observed in GGOs do not progress but persist in the same size or morphology for many years $(4,5)$.

According to the National Comprehensive Cancer Network (NCCN) guidelines for non-small cell lung cancers (NSCLCs; Version 3.2018), pure GGO or partsolid GGO with solid component $\leq 5 \mathrm{~mm}$ is not an indication for surgery. Other guidelines about sub-solid nodules were not different from the NCCN guideline $(6,7)$. Pure GGO or part-solid GGO with solid component 
$\leq 5 \mathrm{~mm}$ were likely to be non-invasive or minimally invasive lung cancer. Therefore, it seems that there is no problem in performing only regular follow-ups without surgical resection of such lesions. However, because of the presence of invasive adenocarcinoma in some of the lung cancers presenting as pure GGO or part-solid GGO with solid component $\leq 5 \mathrm{~mm}$ on chest CT, such lesions may be more appropriate for surgical resection than for regular followup (8-10). Furthermore, the development of minimally invasive surgery has reduced the risk of surgery, so surgical treatment is no longer a major risk to the patient.

Therefore, if invasive adenocarcinoma can be found among pure GGO or part-solid GGO with solid component $\leq 5 \mathrm{~mm}$, it may be easier to determine in which cases surgical treatment should be appropriate. The aim of this study was to determine the predictors of invasive adenocarcinoma in pure GGO or part-solid GGO with solid component $\leq 5 \mathrm{~mm}$ on chest CT. The goal was to determine whether to perform surgery immediately in some lesions showing persistent pure GGO or part-solid GGO with solid component $\leq 5 \mathrm{~mm}$ on chest $\mathrm{CT}$.

\section{Methods}

\section{Patients}

From January 2010 to December 2017, 1,630 consecutive patients at Seoul St. Mary's Hospital in Korea were diagnosed with lung cancer and underwent therapeutic surgical resection. The surgical procedures included both sublobar resection (wedge resection or segmentectomy) and lobectomy, because invasive adenocarcinoma or AIS/MIA can be diagnosed regardless of the surgical procedure. Of those patients, 203 were diagnosed as clinical adenocarcinoma in situ (cAIS) or clinical minimally invasive adenocarcinoma (cMIA) identified on chest CT. Preoperative chemo- or radiotherapy was not administered in any patient. cAIS and cMIA were surgically excised and classified into pathological AIS, pathological MIA, and invasive adenocarcinoma according to the results of the final histologic examination. Pathological AIS (pAIS) and MIA (pMIA) were combined into a single noninvasive adenocarcinoma group (pAIS/MIA) and compared with the invasive adenocarcinoma group. Predictive factors for invasive adenocarcinoma of all patients were analyzed. This study was approved by the institutional review board of Seoul St. Mary's Hospital, The Catholic University of Korea (No. KC18RESI0114).

\section{Preoperative radiologic evaluation and clinical staging}

TNM staging was based on the 8th edition of the TNM classification proposed by the International Association of Study of Lung Cancer (IASLC) (11). Clinical T staging was performed using only nodule size on CT images. Primary lesions were evaluated using thin-section CT images. All chest CT scans were obtained at full inspiration and were retrospectively examined for GGO nodules. All preoperative chest CT imaging was rechecked by two thoracic surgeons who were blinded to the surgical outcome. On a CT scan, GGO was defined as increased hazy opacities in the lung parenchyma with preservation of the bronchial structures and vascular margins (12). The diameter of the tumor (T) was defined as the largest three-dimensional diameter of the lesion on the lung window setting. The diameter of consolidation (C, solid component) on the lung window setting was also measured, and consolidation was defined as an area of increased opacification that completely obscured the underlying bronchial structures and vascular markings. A pure $\mathrm{GGO}$ had a $\mathrm{C} / \mathrm{T}$ ratio of 0 , whereas in a solid nodule, the $\mathrm{C} / \mathrm{T}$ ratio was 1 . A nodule that is part-solid had a $\mathrm{C} / \mathrm{T}$ ratio between 0 and 1 . The definition of clinical AIS and clinical MIA are the same as Tis and T1a (mi) in the 8th edition of the TNM classification (13). This study was performed on GGO-predominant tumors $\leq 3 \mathrm{~cm}$ with a C/ $\mathrm{T}$ ratio $<0.5$. Of the tumors with a $\mathrm{C} / \mathrm{T}$ ratio $<0.5$, pure GGO was defined as cAIS. cMIA was defined as the case when the consolidation diameter was $\leq 5 \mathrm{~mm}$ in the GGOpredominant tumor.

\section{Pathologic staging and histologic evaluation}

When GGO was observed continuously for more than 3 months, the decision to proceed with surgery was made when the size of the GGO lesion exceeded $1 \mathrm{~cm}$, the size of the GGO lesion was $<1 \mathrm{~cm}$ but was increasing, or the GGO had a solid component.

All clinical specimens were examined by a pathology specialist, whose observations were recorded. To describe the histologic patterns of tumors, the occupancy ratio of each histologic component (lepidic, acinar, papillary, micropapillary, and solid) in the total tumor area was measured and recorded semi-quantitatively in $5 \%$ increments according to the 2015 WHO classification of lung tumors (14). AIS and MIA were defined as small $(\leq 3 \mathrm{~cm})$, and solitary adenocarcinomas consisted of lepidic component without invasive component (AIS) or with 
$\leq 5 \mathrm{~mm}$ invasive component (MIA). Invasive adenocarcinomas were classified into one of several subtypes (acinar adenocarcinoma, papillary adenocarcinoma, micropapillary adenocarcinoma, lepidic adenocarcinoma, and others).

\section{Statistical analyses}

Clinicopathological factors of the pAIS/MIA group and invasive adenocarcinoma group were compared in all patients using either student's $t$-test or the Wilcoxon ranksum test for continuous variables, and using the $\chi^{2}$ test or Fisher's exact test for categorical variables. Multivariate logistic regression was used to analyze the predictive factors for invasive adenocarcinoma in all patients. All variables with a $\mathrm{P}<0.1$ in the univariate analysis were entered into a multivariate analysis. Subgroup analysis was also done. In cAIS group and cMIA group, predictive factors for invasive adenocarcinoma were analyzed respectively. A $P$ value $<0.05$ was considered statistically significant. Statistical analyses were performed using SPSS 24.0 software (IBM Corp, Armonk, NY, USA).

\section{Results}

Clinical characteristics and surgical outcomes are presented in Table 1. Mean diameter of the consolidation on chest CT

Table 1 Clinical characteristics of clinical adenocarcinoma in situ (cAIS) or clinical minimally invasive adenocarcinoma (cMIA)

\begin{tabular}{lc}
\hline Variables & Mean $( \pm$ SD) or N $(\%)$ \\
\hline Age $( \pm$ SD) & $59( \pm 10.3)$ \\
Sex & $70(34.5)$ \\
Male & $133(65.5)$ \\
Female & $49(24.1)$ \\
Current or former smoker & $1.4( \pm 1.2)$ \\
Serum CEA level $(\mathrm{ng} / \mathrm{mL})( \pm \mathrm{SD})$ & $0.9( \pm 0.6)$ \\
SUVmax & $96.5( \pm 15.2)$ \\
FEV1 $(\%)( \pm S D)$ & $89.1( \pm 16.6)$ \\
DLCO $(\%)( \pm S D)$ & $66(32.5)$ \\
Presence of past cancer history & $11(5.4)$ \\
Metachronous lung cancer & $55(27.1)$ \\
history & $137(67.5)$ \\
Other cancers &
\end{tabular}

Table 1 (continued)
Table 1 (continued)

\begin{tabular}{|c|c|}
\hline Variables & Mean $( \pm$ SD) or N (\%) \\
\hline \multicolumn{2}{|l|}{ Radiologic features } \\
\hline Presence of synchronous GGO & $26(12.8)$ \\
\hline \multicolumn{2}{|l|}{ Tumor location } \\
\hline Central & $3(1.5)$ \\
\hline Peripheral & $200(98.5)$ \\
\hline \multicolumn{2}{|l|}{ Involved lobe } \\
\hline Right upper & $73(36.0)$ \\
\hline Right middle & $19(9.4)$ \\
\hline Right lower & $38(18.7)$ \\
\hline Left upper & $43(21.2)$ \\
\hline Left lower & $30(14.8)$ \\
\hline $\begin{array}{l}\text { Diameter of the consolidation } \\
(\mathrm{mm})( \pm \mathrm{SD})\end{array}$ & $1.7( \pm 1.8)($ range, 0-4.94) \\
\hline $\begin{array}{l}\text { Diameter of the tumor (GGO) } \\
(\mathrm{mm})( \pm \mathrm{SD})\end{array}$ & $14.5( \pm 5.1)($ range, 3.3-28.3) \\
\hline Presence of pleural retraction & $84(41.4)$ \\
\hline $\mathrm{C} / \mathrm{T}$ ratio & $0.1( \pm 0.1)$ \\
\hline \multicolumn{2}{|l|}{ Surgical procedures } \\
\hline \multicolumn{2}{|l|}{ Operation } \\
\hline Wedge resection & $58(28.6)$ \\
\hline Segmentectomy & $52(25.6)$ \\
\hline Lobectomy & $92(45.3)$ \\
\hline Bilobectomy & $1(0.5)$ \\
\hline VATS & $188(92.6)$ \\
\hline Open thoracotomy & $15(7.4)$ \\
\hline No mediastinal node evaluation & $73(36.0)$ \\
\hline $\begin{array}{l}\text { Mediastinal lymph node } \\
\text { dissection }\end{array}$ & $93(45.8)$ \\
\hline $\begin{array}{l}\text { Mediastinal lymph node } \\
\text { sampling }\end{array}$ & $37(18.2)$ \\
\hline \multicolumn{2}{|l|}{ Surgical outcomes } \\
\hline $\begin{array}{l}\text { Duration of postoperative } \\
\text { hospital stay }\end{array}$ & $5.6( \pm 5.5)$ \\
\hline Complications & $19(9.4)$ \\
\hline Prolonged air leak & 16 \\
\hline Pneumonia & 1 \\
\hline lleus & 1 \\
\hline Cerebral infarction & 1 \\
\hline In-hospital mortality & 0 \\
\hline
\end{tabular}

SD, standard deviation; CEA, carcinoembryonic antigen; SUVmax, maximum standardized uptake value; FEV1, forced expiratory volume in 1 second; DLCO, diffusing capacity for carbon monoxide; GGO, ground glass opacity; C/T ratio, diameter of consolidation/diameter of the tumour ratio; VATS, video-assisted thoracoscopic surgery. 
Table 2 Pathological diagnosis of surgically resected clinical AIS (cTisN0M0) or MIA [cT1a(mi)N0M0]

\begin{tabular}{lc}
\hline Variables & $\mathrm{N}(\%)$ \\
\hline All & 203 \\
AIS & $38(18.7)$ \\
MIA & $110(54.2)$ \\
Invasive adenocarcinoma & $55(27.1)$ \\
Clinical AIS (pure GGO) & 106 \\
AIS & $37(34.9)$ \\
MIA & $50(47.2)$ \\
Invasive adenocarcinoma & $19(17.9)$ \\
Clinical MIA (part-solid GGO) & 97 \\
AIS & $1(1.0)$ \\
MIA & $60(61.9)$ \\
Invasive adenocarcinoma & $36(37.1)$ \\
Invasive adenocarcinoma & 55 \\
Lepidic adenocarcinoma & $21(38.2)$ \\
Acinar adenocarcinoma & $27(49.1)$ \\
Papillary adenocarcinoma & $7(12.7)$ \\
\hline GGO, ground glass opacity; AIS, & \\
\hline
\end{tabular}

GGO, ground glass opacity; AIS, adenocarcinoma in situ (TisNOM0); MIA, Minimally invasive adenocarcinoma [T1a(mi) NOMO].

Table 3 Comparison of pathologic diagnosis between pure GGO and Part-solid GGO

\begin{tabular}{lccc}
\hline Variables & Pure GGO (cAIS) & $\begin{array}{c}\text { Part-solid GGO } \\
\text { (cMIA) }\end{array}$ & P value \\
\hline pAIS/MIA & $87(82.1 \%)$ & $61(62.9 \%)$ & 0.002 \\
Invasive & $19(17.9 \%)$ & $36(37.1 \%)$ & \\
adenocarcinoma & & & \\
\hline
\end{tabular}

GGO, ground glass opacity; AIS, adenocarcinoma in situ (TisNOM0); MIA, Minimally invasive adenocarcinoma [T1a(mi) NOMO].

was $1.7 \mathrm{~mm}$ and mean tumor (GGO) size was $14.5 \mathrm{~mm}$. More than half of the surgical procedures were sublobar resections (wedge resection 28.6\%, segmentectomy $25.6 \%$ ). Most procedures $(92.6 \%)$ were performed with videoassisted thoracoscopic surgery (VATS). Mean duration of the postoperative hospital stay was 5.6 days. There were 19 complications (9.4\%) among the patients. However, all
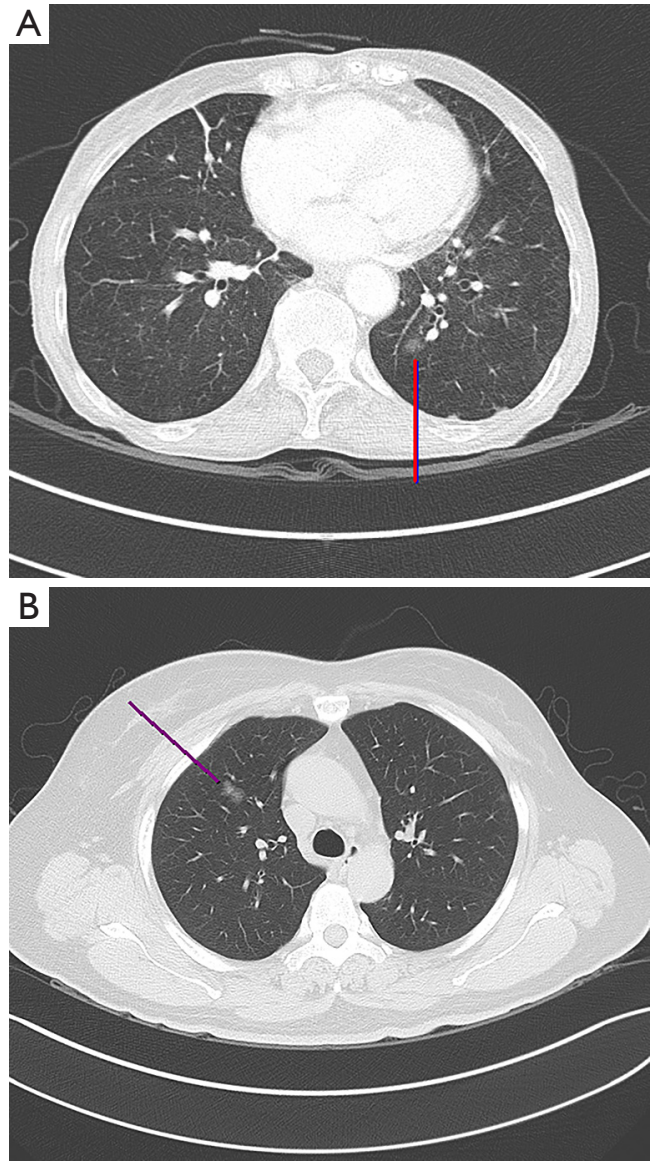

Figure 1 CT images of clinical AIS (A) and MIA (B) diagnosed as invasive adenocarcinoma. AIS, adenocarcinoma in situ; MIA, minimally invasive adenocarcinoma; CT, computed tomography.

complications were resolved during the hospital stay. There was no in-hospital mortality.

The pathological diagnosis of all patients is summarized in Tables 2,3. Although clinical diagnoses of all patients were AIS or MIA, invasive adenocarcinoma was diagnosed in 55 patients (27.1\%) (Figure 1). In the cAIS group, invasive adenocarcinoma was diagnosed in 19 patients (17.9\%). Thirty-six patients (37.1\%) were diagnosed as invasive adenocarcinoma in the cMIA group. The incidence of invasive adenocarcinoma between cAIS and cMIA were statistically different $(\mathrm{P}=0.002)$. Preoperative cAIS or cMIA, and postoperative invasive adenocarcinoma included only three subtypes of invasive adenocarcinoma: acinar adenocarcinoma, lepidic adenocarcinoma, and papillary adenocarcinoma. 
Table 4 Comparison of preoperative factors between pathological AIS/MIA and invasive adenocarcinoma in patients with clinical AIS or clinical MIA

\begin{tabular}{|c|c|c|c|}
\hline Variables & $\begin{array}{l}\mathrm{pAIS} / \mathrm{MIA} \\
(\mathrm{n}=148)\end{array}$ & $\begin{array}{c}\text { Invasive } \\
\text { adenocarcinoma } \\
(\mathrm{n}=55)\end{array}$ & $\begin{array}{c}P \\
\text { value }\end{array}$ \\
\hline Age $( \pm S D)$ & $59.9( \pm 10.2)$ & $58.7( \pm 10.4)$ & 0.440 \\
\hline Sex & & & 0.188 \\
\hline Male & $55(37.2 \%)$ & $15(27.3 \%)$ & \\
\hline Female & $93(62.8 \%)$ & $40(72.7 \%)$ & \\
\hline Current or former smoker & $35(23.6 \%)$ & $14(25.5 \%)$ & 0.789 \\
\hline $\begin{array}{l}\text { Serum CEA level }(\mathrm{ng} / \mathrm{mL}) \\
( \pm \mathrm{SD})\end{array}$ & $1.5( \pm 1.2)$ & $1.3( \pm 1.3)$ & 0.423 \\
\hline SUVmax $( \pm$ SD) & $0.9( \pm 0.6)$ & $1.0( \pm 0.7)$ & 0.525 \\
\hline FEV1 (\%) ( \pm SD) & $95.7( \pm 15.5)$ & $98.5( \pm 14.6)$ & 0.260 \\
\hline DLCO (\%) ( \pm SD) & $88.3( \pm 17.6)$ & $90.9( \pm 13.7)$ & 0.342 \\
\hline $\begin{array}{l}\text { Presence of past cancer } \\
\text { history }\end{array}$ & $54(36.5 \%)$ & $12(21.8 \%)$ & 0.047 \\
\hline $\begin{array}{l}\text { Presence of synchronous } \\
\text { GGO }\end{array}$ & $21(14.2 \%)$ & $5(9.1 \%)$ & 0.334 \\
\hline Central location & $2(1.4 \%)$ & $1(1.8 \%)$ & 1.000 \\
\hline Involved lobe & & & 0.652 \\
\hline Right upper & $57(38.5 \%)$ & $16(29.1 \%)$ & \\
\hline Right middle & $14(9.5 \%)$ & $5(9.1 \%)$ & \\
\hline Right lower & $28(18.9 \%)$ & $10(18.2 \%)$ & \\
\hline Left upper & $28(18.9 \%)$ & $15(27.3 \%)$ & \\
\hline Left lower & $21(14.2 \%)$ & $9(16.4 \%)$ & \\
\hline $\begin{array}{l}\text { Diameter of the } \\
\text { consolidation }(\mathrm{mm})( \pm \mathrm{SD})\end{array}$ & $1.4( \pm 1.8)$ & $2.4( \pm 1.9)$ & 0.001 \\
\hline $\begin{array}{l}\text { Diameter of the tumor } \\
(\mathrm{GGO})(\mathrm{mm})( \pm \mathrm{SD})\end{array}$ & $14.0( \pm 4.7)$ & $16.0( \pm 5.7)$ & 0.018 \\
\hline $\begin{array}{l}\text { Presence of pleural } \\
\text { retraction }\end{array}$ & $58(39.2 \%)$ & $26(47.3 \%)$ & 0.299 \\
\hline
\end{tabular}

SD, standard deviation; CEA, carcinoembryonic antigen; SUVmax, maximum standardized uptake value; FEV1, forced expiratory volume in 1 second; DLCO, diffusing capacity for carbon monoxide; GGO, ground glass opacity.

\section{Predictive factors for invasive adenocarcinoma in all patients}

We compared clinical factors between the pAIS/MIA and invasive adenocarcinoma groups in all patients
(Table 4). There were no differences between the two groups except for the presence of past cancer history, diameter of consolidation, and tumor diameter. Logistic regression analysis was used to determine the predictive factors for invasive adenocarcinoma in all patients. In univariate analysis, past cancer history, diameter of consolidation, and tumor diameter had $\mathrm{P}$ values $<0.1$. These variables were entered into the multivariate model [Table 5 (A)]. Diameters of the consolidation and tumor were confirmed to be significant predictive factors for invasive adenocarcinoma in a multivariate analysis [hazard ratio $(\mathrm{HR})=1.296, \mathrm{P}=0.003$; $\mathrm{HR}=1.068, \mathrm{P}=0.048$, respectively] [Table 5 (B)].

\section{Predictive factors for invasive adenocarcinoma in subgroups}

We analyzed predictive factors for invasive adenocarcinoma in cAIS group and cMIA group respectively.

In the cAIS group, specific variables identified as significant $(\mathrm{P}<0.1)$ by univariate analysis included presence of past cancer history, tumor diameter, and presence of pleural retraction. These variables were entered into the multivariate model. Only tumor diameter was significantly predictive of invasive adenocarcinoma $(H R=1.166$, $\mathrm{P}=0.006$ ) (Table 6).

On the other hand, there was no significant predictive factor for invasive adenocarcinoma in the cMIA group on logistic regression analysis. Even the diameter of the consolidation was not a significant predictor for invasive adenocarcinoma (Table 7). In fact, the consolidation size was not measured in cAIS, but only in cMIA. Consolidation size was a significant predictor in all patients, but was not a significant predictor in cMIA. In other words, consolidation rather than consolidation size was a predictor in all patients. Therefore, the presence of consolidation (solid component) was a significant predictor in multivariate analysis of all the patients by changing the diameter of the consolidation to the presence of solid component $(\mathrm{HR}=2.573, \mathrm{P}=0.005$ ) [Table $5(\mathrm{C})$ ].

We created a scatter plot to see how the pure GGO diameters were distributed in the pAIS, pMIA, and invasive adenocarcinoma groups (Figure 2). All tumors in the invasive adenocarcinoma group were $\geq 1.0 \mathrm{~cm}$. Therefore, invasive adenocarcinoma was not detected in cases in which the diameter of pure GGO was $<1.0 \mathrm{~cm}$. On the other hand, in case of part-solid GGO, the size distribution of invasive adenocarcinoma varied (range, $5-26.5 \mathrm{~mm}$ ). Although partsolid GGO has a very small size, it may be diagnosed as 
Table 5 Predictive factors for invasive adenocarcinoma on logistic regression analysis in patients with clinical AIS or MIA

\begin{tabular}{|c|c|c|c|}
\hline Variables & HR & $95 \% \mathrm{Cl}$ & $P$ value \\
\hline \multicolumn{4}{|l|}{ (A) Univariate analysis } \\
\hline Age & 0.988 & $0.959-1.018$ & 0.438 \\
\hline Sex (male) & 0.634 & $0.321-1.252$ & 0.190 \\
\hline Current or former smoker & 1.102 & $0.539-2.255$ & 0.789 \\
\hline Serum CEA level & 0.890 & $0.670-1.183$ & 0.424 \\
\hline SUVmax & 1.220 & $0.663-2.246$ & 0.523 \\
\hline FEV1 (\%) & 1.012 & $0.991-1.033$ & 0.260 \\
\hline DLCO (\%) & 1.009 & $0.990-1.029$ & 0.341 \\
\hline $\begin{array}{l}\text { Presence of past cancer } \\
\text { history }\end{array}$ & 0.486 & $0.236-1.000$ & 0.050 \\
\hline Presence of synchronous GGO & 0.605 & $0.216-1.692$ & 0.338 \\
\hline Central location & 1.343 & $0.119-15.109$ & 0.811 \\
\hline Involved lobe & & & 0.657 \\
\hline Right upper (reference) & 1 & & \\
\hline Right middle & 1.272 & $0.398-4.067$ & 0.685 \\
\hline Right lower & 1.272 & $0.512-3.162$ & 0.604 \\
\hline Left upper & 1.908 & $0.826-4.408$ & 0.130 \\
\hline Left lower & 1.527 & $0.586-3.978$ & 0.386 \\
\hline Diameter of consolidation (mm) & 1.327 & $1.119-1.574$ & 0.001 \\
\hline Diameter of tumor (GGO) & 1.083 & $1.019-1.152$ & 0.011 \\
\hline Presence of pleural retraction & 1.391 & $0.746-2.596$ & 0.300 \\
\hline \multicolumn{4}{|l|}{ (B) Multivariate analysis } \\
\hline $\begin{array}{l}\text { Presence of past cancer } \\
\text { history }\end{array}$ & 0.605 & $0.284-1.287$ & 0.192 \\
\hline $\begin{array}{l}\text { Diameter of the consolidation } \\
(\mathrm{mm})\end{array}$ & 1.296 & $1.090-1.542$ & 0.003 \\
\hline Diameter of the tumor (GGO) & 1.068 & $1.001-1.139$ & 0.048 \\
\hline \multicolumn{4}{|l|}{ (C) Multivariate analysis } \\
\hline Previous cancer history & 0.592 & $0.279-1.258$ & 0.173 \\
\hline Presence of solid component & 2.573 & $1.333-4.967$ & 0.005 \\
\hline Diameter of tumor (GGO) & 1.071 & $1.004-1.143$ & 0.037 \\
\hline
\end{tabular}

$\mathrm{HR}$, hazard ratio; $\mathrm{Cl}$, confidence interval; CEA, carcinoembryonic antigen; SUVmax, maximum standardized uptake value; FEV1, forced expiratory volume in 1 second; DLCO, diffusing capacity for carbon monoxide; GGO, ground glass opacity. invasive adenocarcinoma (Figure 3).

\section{Discussion}

Persistent GGO is most likely lung adenocarcinoma. Particularly, GGO may be considered to reflect the lepidic component (15). Saji et al. (16) reported that the size of a GGO and its solid component are correlated with the pathologic size of a tumor and its invasive component (16). Therefore, pure GGO is likely to be AIS composed of $100 \%$ lepidic component. Part-solid GGO is more likely to be MIA when the solid component size is $<5 \mathrm{~mm}$ (17). This is reflected in the latest (8th edition) of the TNM staging and is the same as defining the clinical TisNOM0 and clinical T1a (mi) NOM0 (13). Therefore, these lesions are considered to be non- or minimally-invasive, so they are often followed-up without immediate surgery. This is why the NCCN guideline recommends only routine follow-up for such GGO lesions.

However, there is a discrepancy between clinical and pathologic stages. Nodal upstaging as well as upstage of $\mathrm{T}$ is often found after surgery. Thus, the clinical stage through preoperative imaging examination cannot be unconditionally confirmed (10,18-21). Clinical AIS (Tis) and MIA [T1a (mi)] are often diagnosed as pAIS or pMIA after surgery, but they are sometimes diagnosed as invasive adenocarcinoma $(8,22,23)$. Heidinger et al. (24) reported that pure GGOs in CT images were ultimately diagnosed as invasive adenocarcinoma in $16 \%$ of patients. In this study, $17.9 \%$ of patients who had a pure GGO were diagnosed as invasive adenocarcinoma. This was similar to the incidence of invasive adenocarcinoma found among surgically resected pure GGOs. Although lepidic components present as GGO on imaging studies, acinar or papillary components, which are invasive components, can sometimes be seen as GGO on CT images $(8,10,17)$. In this study, invasive adenocarcinoma was diagnosed in $27.1 \%$ of all cAIS or cMIA. Moreover, all invasive components were acinar or papillary patterns. Therefore, invasive adenocarcinoma including acinar or papillary component can be misdiagnosed as AIS or MIA in preoperative chest CT. Of course, invasive adenocarcinoma needs immediate surgical treatment.

In this study, large tumor size and the presence of solid component were the significant predictors for invasive 
Table 6 Predictive factors for invasive adenocarcinoma on logistic regression analysis in patients with clinical AIS only (pure GGO)

\begin{tabular}{|c|c|c|c|}
\hline Variables & $\mathrm{HR}$ & $95 \% \mathrm{Cl}$ & $\begin{array}{c}\mathrm{P} \\
\text { value }\end{array}$ \\
\hline \multicolumn{4}{|l|}{ Univariate analysis } \\
\hline Age & 0.991 & $0.948-1.037$ & 0.704 \\
\hline Sex (male) & 1.403 & $0.517-3.807$ & 0.506 \\
\hline Current or former smoker & 1.451 & $0.490-4.292$ & 0.502 \\
\hline CEA & 1.099 & $0.805-1.500$ & 0.552 \\
\hline SUVmax & 1.558 & $0.688-3.530$ & 0.288 \\
\hline FEV1 (\%) & 1.017 & $0.985-1.050$ & 0.308 \\
\hline DLCO (\%) & 1.009 & $0.978-1.040$ & 0.575 \\
\hline Previous cancer history & 0.279 & $0.076-1.028$ & 0.055 \\
\hline $\begin{array}{l}\text { Presence of synchronous } \\
\text { GGO }\end{array}$ & 0.900 & $0.233-3.481$ & 0.879 \\
\hline Involved lobe & & & 0.429 \\
\hline Right upper (reference) & 1 & & \\
\hline Right middle & 1.850 & $0.171-20.029$ & 0.613 \\
\hline Right lower & 2.176 & $0.486-9.756$ & 0.310 \\
\hline Left upper & 3.964 & $0.971-16.184$ & 0.055 \\
\hline Left lower & 2.643 & $0.580-12.038$ & 0.209 \\
\hline Radiologic tumor size (mm) & 1.186 & $1.070-1.314$ & 0.001 \\
\hline Radiologic pleural retraction & 4.063 & $1.449-11.392$ & 0.008 \\
\hline \multicolumn{4}{|l|}{ Multivariate analysis } \\
\hline $\begin{array}{l}\text { Presence of past cancer } \\
\text { history }\end{array}$ & 0.359 & $0.088-1.470$ & 0.154 \\
\hline Diameter of the tumor (GGO) & 1.166 & $1.045-1.301$ & 0.006 \\
\hline Presence of pleural retraction & 2.711 & $0.895-8.214$ & 0.078 \\
\hline
\end{tabular}

$\mathrm{HR}$, hazard ratio; $\mathrm{Cl}$, confidence interval; CEA, carcinoembryonic antigen; SUVmax, maximum standardized uptake value; FEV1, Forced expiratory volume in 1 second; DLCO, diffusing capacity for carbon monoxide; GGO, ground glass opacity.

adenocarcinoma in cAIS or cMIA on chest CT. The size distribution of invasive adenocarcinoma in pure GGO showed no invasive adenocarcinoma $<10 \mathrm{~mm}$. Therefore, in the case of pure GGO, the routine follow-up will not be a problem when the tumor size is $<10 \mathrm{~mm}$. On the other hand, in the case of part-solid GGOs, five cases were diagnosed as invasive adenocarcinoma even if the size was $<10 \mathrm{~mm}$. Therefore, part-solid GGO may require surgical
Table 7 Predictive factors for invasive adenocarcinoma on logistic regression analysis in patients with clinical MIA only (part-solid GGO)

\begin{tabular}{|c|c|c|c|}
\hline Variable & $\mathrm{HR}$ & $95 \% \mathrm{Cl}$ & $\begin{array}{c}\mathrm{P} \\
\text { value }\end{array}$ \\
\hline \multicolumn{4}{|l|}{ Univariate analysis } \\
\hline Age & 0.974 & $0.932-1.018$ & 0.241 \\
\hline Sex (male) & 0.381 & $0.137-1.060$ & 0.065 \\
\hline Current of former smoker & 0.959 & $0.358-2.573$ & 0.934 \\
\hline CEA & 0.648 & $0.380-1.104$ & 0.110 \\
\hline SUVmax & 0.831 & $0.323-2.138$ & 0.701 \\
\hline FEV1 (\%) & 1.006 & $0.978-1.035$ & 0.683 \\
\hline DLCO (\%) & 1.009 & $0.983-1.035$ & 0.499 \\
\hline Previous cancer history & 0.737 & $0.291-1.865$ & 0.519 \\
\hline Presence of Synchronous GGO & 0.539 & $0.103-2.826$ & 0.465 \\
\hline Involved lobe & & & 0.983 \\
\hline Right upper (reference) & 1 & & \\
\hline Right middle & 0.741 & $0.187-2.939$ & 0.670 \\
\hline Right lower & 0.909 & $0.267-3.096$ & 0.879 \\
\hline Left upper & 1.071 & $0.356-3.224$ & 0.902 \\
\hline Left lower & 1.190 & $0.308-4.604$ & 0.801 \\
\hline $\begin{array}{l}\text { Diameter of the consolidation } \\
(\mathrm{mm})\end{array}$ & 1.324 & $0.821-2.135$ & 0.250 \\
\hline Diameter of the tumor (GGO) & 1.009 & $0.928-1.097$ & 0.832 \\
\hline Presence of pleural retraction & 0.496 & $0.215-1.145$ & 0.100 \\
\hline
\end{tabular}

excision even if the size is small. In this study, invasive adenocarcinoma was diagnosed in $37.1 \%$ of part-solid GGO, which were considered cMIA. In other words, the incidence of invasive adenocarcinoma is relatively high, so it is doubtful whether all of the part-solid GGOs can be regarded as less invasive tumors.

Surgery is performed at our institution when (I) the size of a persistent GGO is $>1 \mathrm{~cm}$, (II) the GGO is increasing in size, or (III) a part-solid GGO is persistent for several months, regardless of its size. Recently, the development of minimally invasive surgery has reduced the burden of surgery for many patients. VATS is the most preferred method for surgical resection of GGO lesions. In this study, 


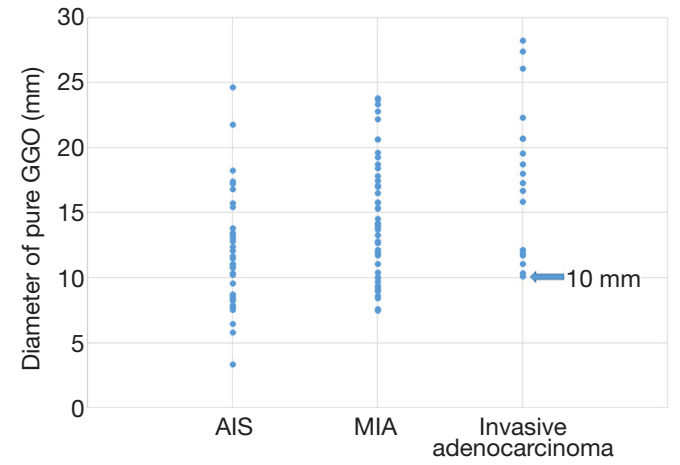

Figure 2 Scatter plot of pathologic diagnosis and diameter of pure GGO. All tumors in the invasive adenocarcinoma group were $10 \mathrm{~mm}$ or larger. GGO, ground glass opacity.

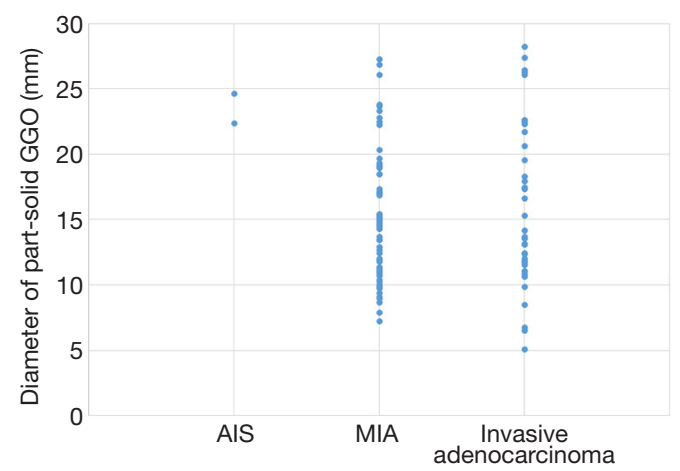

Figure 3 Scatter plot of pathologic diagnosis and diameter of partsolid GGO. Small tumors less than $10 \mathrm{~mm}$ were also diagnosed as invasive adenocarcinoma. GGO, ground glass opacity.

most surgeries were performed using VATS. The incidence rate of postoperative complications was low (9.4\%). In fact, most complications were prolonged air leak ( $>5$ days) and were resolved within 2 weeks. The incidence rate of postoperative complication of VATS wedge resection was very low. In addition, the extent of surgical resection may be reduced for GGO lesions. For example, a number of studies have reported good prognosis when sublobar resection is applied to peripheral pure GGO or part-solid GGO (24-26). We have also reported that the prognosis after limited resection is fairly good even if the lesion presenting as GGO on chest CT is diagnosed as invasive adenocarcinoma postoperatively (19). Therefore, if the minimally invasive surgery and sublobar resection are performed in an appropriate manner to perform surgery on GGO lesions, there will be no missed invasive adenocarcinoma without significant surgical burden on the patient.
This study had several limitations that should be considered. First, we used a retrospective study design. Second, we obtained the data from a single institution and the number of cases was relatively small. However, all data in this study are recent, since 2010, and since diagnosis was performed according to the same protocol, the bias should be relatively low. Future studies with more patients may provide more accurate results. Third, our study was restricted to surgical patients. In fact, it is difficult to apply the results of this study to all GGO cases because many of the GGO lesions are not surgically resected. However, it is meaningful if the lesion is growing GGO, persistent partsolid, or persistent pure GGO ( $\geq 1 \mathrm{~cm})$. In fact, our protocol for surgical treatment of GGO lesions revealed that those GGO lesions were always adenocarcinoma. Therefore, this study can be applied to GGO lesions that may consider surgery.

In conclusion, a persistent pure GGO or part-solid GGO with a solid component $\leq 5 \mathrm{~mm}$ is usually considered as being a non-invasive or minimally invasive lesion, while a tumor with a larger size or a GGO with solid component is more likely to be an invasive adenocarcinoma. Furthermore, immediate surgical removal of such lesions does not appear to be an unreasonable treatment, because the risk of minimally invasive surgery is not high. Therefore, immediate surgery for patients with a persistent large GGO or a part-solid GGO is a good option for the treatment of lung cancer when compared with just follow-up. Further study and additional data are needed for generalize our results.

\section{Acknowledgements}

None.

\section{Footnote}

Conflicts of Interest: The authors have no conflicts of interest to declare.

Ethical Statement: This study was approved by the institutional review board of Seoul St. Mary's Hospital, The Catholic University of Korea (No. KC18RESI0114).

\section{References}

1. Aberle DR, Adams AM, Berg CD, et al. Reduced lungcancer mortality with low-dose computed tomographic 
screening. N Engl J Med 2011;365:395-409.

2. Henschke CI, Yankelevitz DF, Mirtcheva R, et al. CT screening for lung cancer: frequency and significance of part-solid and nonsolid nodules. AJR Am J Roentgenol 2002;178:1053-7.

3. Kim HY, Shim YM, Lee KS, et al. Persistent pulmonary nodular ground-glass opacity at thin-section CT: histopathologic comparisons. Radiology 2007;245:267-75.

4. Kakinuma R, Noguchi M, Ashizawa K, et al. Natural History of Pulmonary Subsolid Nodules: A Prospective Multicenter Study. J Thorac Oncol 2016;11:1012-28.

5. Cho J, Kim ES, Kim SJ, et al. Long-Term Follow-up of Small Pulmonary Ground-Glass Nodules Stable for 3 Years: Implications of the Proper Follow-up Period and Risk Factors for Subsequent Growth. J Thorac Oncol 2016;11:1453-9.

6. MacMahon H, Naidich DP, Goo JM, et al. Guidelines for Management of Incidental Pulmonary Nodules Detected on CT Images: From the Fleischner Society 2017. Radiology 2017;284:228-43.

7. Callister ME, Baldwin DR, Akram AR, et al. British Thoracic Society guidelines for the investigation and management of pulmonary nodules. Thorax 2015;70 Suppl 2:ii1-54.

8. Moon Y, Sung SW, Lee KY, et al. Pure ground-glass opacity on chest computed tomography: predictive factors for invasive adenocarcinoma. J Thorac Dis 2016;8:1561-70.

9. Liu LH, Liu M, Wei R, et al. CT Findings of Persistent Pure Ground Glass Opacity: Can We Predict the Invasiveness? Asian Pac J Cancer Prev 2015;16:1925-8.

10. Moon Y, Sung SW, Lee KY, et al. Clinicopathological characteristics and prognosis of non-lepidic invasive adenocarcinoma presenting as ground glass opacity nodule. J Thorac Dis 2016;8:2562-70.

11. Goldstraw P, Chansky K, Crowley J, et al. The IASLC Lung Cancer Staging Project: Proposals for Revision of the TNM Stage Groupings in the Forthcoming (Eighth) Edition of the TNM Classification for Lung Cancer. J Thorac Oncol 2016;11:39-51.

12. Hansell DM, Bankier AA, MacMahon H, et al. Fleischner Society: glossary of terms for thoracic imaging. Radiology 2008;246:697-722.

13. Travis WD, Asamura H, Bankier AA, et al. The IASLC Lung Cancer Staging Project: Proposals for Coding T Categories for Subsolid Nodules and Assessment of Tumor Size in Part-Solid Tumors in the Forthcoming Eighth Edition of the TNM Classification of Lung Cancer. J
Thorac Oncol 2016;11:1204-23.

14. Travis WD, Brambilla E, Nicholson AG, et al. The 2015 World Health Organization Classification of Lung Tumors: Impact of Genetic, Clinical and Radiologic Advances Since the 2004 Classification. J Thorac Oncol 2015;10:1243-60.

15. Eguchi T, Kadota K, Park BJ, et al. The new IASLCATS-ERS lung adenocarcinoma classification: what the surgeon should know. Semin Thorac Cardiovasc Surg 2014;26:210-22.

16. Saji H, Matsubayashi J, Akata S, et al. Correlation between whole tumor size and solid component size on highresolution computed tomography in the prediction of the degree of pathologic malignancy and the prognostic outcome in primary lung adenocarcinoma. Acta Radiol 2015;56:1187-95.

17. Wilshire CL, Louie BE, Manning KA, et al. Radiologic Evaluation of Small Lepidic Adenocarcinomas to Guide Decision Making in Surgical Resection. Ann Thorac Surg 2015;100:979-88.

18. Moon Y, Kim KS, Lee KY, et al. Clinicopathologic Factors Associated With Occult Lymph Node Metastasis in Patients With Clinically Diagnosed N0 Lung Adenocarcinoma. Ann Thorac Surg 2016;101:1928-35.

19. Moon Y, Lee KY, Park JK. The prognosis of invasive adenocarcinoma presenting as ground-glass opacity on chest computed tomography after sublobar resection. J Thorac Dis 2017;9:3782-92.

20. Moon Y, Sung SW, Namkoong M, et al. The effectiveness of mediastinal lymph node evaluation in a patient with ground glass opacity tumor. J Thorac Dis 2016;8:2617-25.

21. Moon Y, Park JK, Lee KY, et al. Consolidation/Tumor Ratio on Chest Computed Tomography as Predictor of Postoperative Nodal Upstaging in Clinical T1N0 Lung Cancer. World J Surg 2018;42:2872-8.

22. Son JY, Lee HY, Kim JH, et al. Quantitative CT analysis of pulmonary ground-glass opacity nodules for distinguishing invasive adenocarcinoma from non-invasive or minimally invasive adenocarcinoma: the added value of using iodine mapping. Eur Radiol 2016;26:43-54.

23. Jin X, Zhao SH, Gao J, et al. CT characteristics and pathological implications of early stage (T1N0M0) lung adenocarcinoma with pure ground-glass opacity. Eur Radiol 2015;25:2532-40.

24. Yoshida J, Nagai K, Yokose T, et al. Limited resection trial for pulmonary ground-glass opacity nodules: fifty-case experience. J Thorac Cardiovasc Surg 2005;129:991-6.

25. Cho JH, Choi YS, Kim J, et al. Long-term outcomes 
of wedge resection for pulmonary ground-glass opacity nodules. Ann Thorac Surg 2015;99:218-22.

26. Moon Y, Lee KY, Moon SW, et al. Sublobar Resection Margin Width Does Not Affect Recurrence of Clinical
N0 Non-small Cell Lung Cancer Presenting as GGOPredominant Nodule of $3 \mathrm{~cm}$ or Less. World J Surg 2017;41:472-9.

Cite this article as: Moon Y, Park JK, Lee KY, Ahn S, Shin J. Predictive factors for invasive adenocarcinoma in patients with clinical non-invasive or minimally invasive lung cancer. J Thorac Dis 2018;10(11):6010-6019. doi: 10.21037/ jtd.2018.10.83 